Article

\title{
Intramolecular Chain Hydrosilylation of Alkynylphenylsilanes Using a Silyl Cation as a Chain Carrier
}

\author{
Hidekazu Arii ${ }^{1, *}$, Kenichi Nakabayashi ${ }^{1}$, Kunio Mochida ${ }^{2}$ and Takayuki Kawashima ${ }^{3, *}$ \\ 1 Faculty of Education, University of Miyazaki, 1-1 Gakuen Kibanadai Nishi, Miyazaki, 889-2192 Miyazaki, \\ Japan; nakabys@cc.miyazaki-u.ac.jp \\ 2 Department of Chemistry, Gakushuin University, 1-5-1 Mejiro, Toshima-ku, 171-8588 Tokyo, Japan; \\ kunio.mochida@gakushuin.ac.jp \\ 3 Graduate School of Science and Technology, Gunma University, 1-5-1 Tenjin-cho, Kiryu, \\ 376-8515 Gunma, Japan \\ * Correspondence: hidekazu.arii@cc.miyazaki-u.ac.jp (H.A.); kawashima.t@gunma-u.ac.jp (T.K.); \\ Tel.: +81-985-58-7499 (H.A.); +81-277-30-1670 (T.K.); Fax: +81-985-58-7499 (H.A.); +81-277-30-1202 (T.K.)
}

Academic Editor: Mitsuo Kira

Received: 9 July 2016; Accepted: 27 July 2016; Published: 1 August 2016

\begin{abstract}
Diorganyl[2-(trimethylsilylethynyl)phenyl]silanes 1a-c and methyl-substituted phenylsilanes $\mathbf{1 d}$ and 1e were treated with a small amount of trityl tetrakis(pentafluorophenyl)borate (TPFPB) as an initiator in benzene to afford the corresponding benzosiloles $(\mathbf{2 a}-\mathbf{e})$ in moderate to good yields. However, no reaction was observed for the reaction using [2-(1-hexynyl)phenyl]diisopropylsilane If. The methyl substituent was tolerated under the reaction conditions and increased the yield of the corresponding benzosilole depending on the substitution position. From the result using 1f, the current reaction was found to require the trimethylsilyl group, which can stabilize intermediary alkenyl carbocations by the $\beta$-silyl effect. The current reaction can be considered an intramolecular chain hydrosilylation of alkynylarylsilanes involving silyl cations as chain carriers. Therefore, the silyl cations generated by hydride abstraction from hydrosilanes 1 with the trityl cation causes intramolecular electrophilic addition to the $\mathrm{C}-\mathrm{C}$ triple bond to form ethenyl cations, which abstract a hydride from 1 to afford benzosiloles 2 with the regeneration of the silyl cations.
\end{abstract}

Keywords: benzosilole; silyl cation; hydrosilylation; metal free

\section{Introduction}

A benzosilole is an attractive compound due to its emission property and potential use as of optical materials [1-4]. These features are associated with the low-lying LUMOs of the siloles, which originate from orbital interaction between the $\sigma^{*}$ orbital of the silylene moiety and the $\pi^{*}$ orbital of the butadiene moiety [5]. The popular synthetic routes to benzo- and dibenzosiloles involve intra- and intermolecular cyclization reactions with transition metal catalysts [6-9], and the use of a chiral supporting ligand enables the synthesis of siloles with a chiral silicon center $[10,11]$. In particular, direct $\mathrm{Si}-\mathrm{C}$ or $\mathrm{C}-\mathrm{H}$ activation is a powerful method that does not require an activated functional group on the aromatic ring [12-17]. In non-transition metal systems, 2-ethynylphenylsilane derivatives have been cyclized to afford the corresponding benzosiloles using various reactants, such as lithium naphthalenide [18], Lewis acids [19,20] and KH (Equation (1)) [21]. The radical reaction that is initiated by tert-butylhydroperoxide (TBHP) resulted in the formation of dibenzosiloles from (2-biphenyl)diphenylsilanes (Equation (2)) [22]. The uses of $\mathrm{KH}$ and TBHP produce the corresponding active silicon species (i.e., a pentacoordinated hydridosilicate and a silyl radical, 
respectively). We have synthesized dibenzosilole by a sila-Friedel-Crafts reaction mediated by a silyl cation (Equation (3)) [23,24].
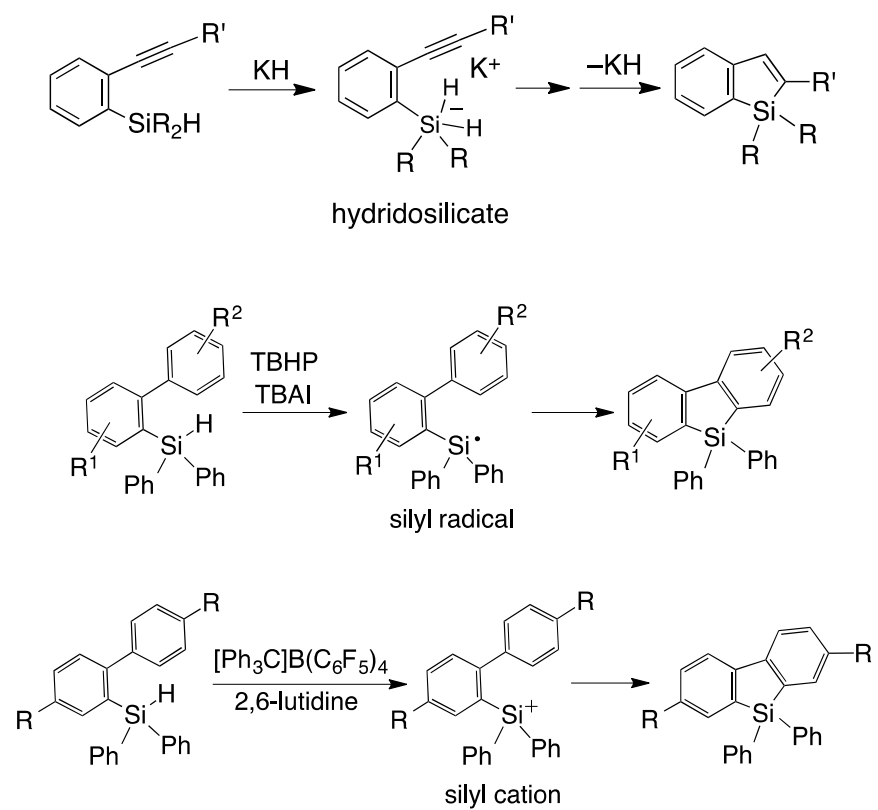

We have extended silyl cation chemistry to the synthesis of silacyclic compounds and reported the synthesis of 1,2-dihydro-2-silanaphthalenes using alkynes $[25,26]$. The intramolecular addition of a silyl cation toward a C-C triple bond rather than an intermolecular one is expected to lead to a benzosilole by an appropriate reaction of the resulting ethenyl carbocation. Herein, we report the intramolecular chain hydrosilylation of diorganyl[2-(trimethylsilylethynyl)phenyl]silanes with trityl tetrakis(pentafluorophenyl)borate (TPFPB) as an initiator.

\section{Results and Discussion}

Diorganyl[2-(trimethylsilylethynyl)phenyl]silanes 1a-c were reacted with a small amount (1-4 mol \%) of TPFPB in benzene to afford the corresponding benzosiloles $2 \mathrm{a}-\mathrm{c}$ in low to moderate yields (Table 1, entries 1-3). The intramolecular chain hydrosilylation of 1 was achieved using TPFPB as an initiator. However, this reaction was accompanied with the formation of unidentified oligomers, which appear to be formed by silyl cation-induced alkyne polymerization. The amount of TPFPB and solvent were optimized using $\mathbf{1 b}$, and therefore, $1 \mathrm{~mol} \%$ TPFPB in benzene was determined to provide the best yield of $75 \%$ (Table 1 , entry 5 ). The conversion of $\mathbf{1} \mathbf{b}$ to silole $\mathbf{2} \mathbf{b}$ required a longer reaction time (30 min in $1 \mathrm{~mol} \%$ TPFPB (Table 1, entry 5) compared to $15 \mathrm{~min}$ in $3 \mathrm{~mol} \%$ TPFPB (entry 4 ) and $5 \mathrm{~min}$ in $4 \mathrm{~mol} \%$ TPFPB (entry 2)) with a decrease in the dose of the trityl cation initiator. However, the yield of $\mathbf{2 b}$ increased from $61 \%$ to $75 \%$ due to the preference of the desired hydrosilylation over the competing oligomerization. The syntheses of benzosiloles from 2-alkynylphenylsilane derivatives in transition metal-free systems have been achieved using $\mathrm{AlCl}_{3}$ and $\mathrm{KH}$ to activate the $\mathrm{Si}-\mathrm{H}$ and /or C-C triple bonds and to generate the pentacoordinated hydridosilicate, respectively $[19,21]$. In this system, the silyl cation plays an important role in the reaction and promotes the hydrosilylation by electrophilic addition to the $\mathrm{C}-\mathrm{C}$ triple bond.

The scope and limitations of the intramolecular chain hydrosilylation of $\mathbf{1}$ are summarized as follows. Under the optimized conditions using $1 \mathrm{~mol} \%$ of TPFPB, the isolated yields of $\mathbf{2 b}$ and $\mathbf{2 c}$ were increased (entries 5 and 10) even though the yield of 2a bearing the sterically smaller methyl groups on the silicon center was barely affected by the dose of initiator (entry 9). The reactions using methyl-substituted silanes $\mathbf{1 d}$ and $\mathbf{1 e}$ also afforded the corresponding benzosiloles $\mathbf{2} \mathbf{d}$ and $\mathbf{2 e}$ in $72 \%$ and $81 \%$ yields, respectively (entries 11 and 12 ). It is most likely that the relatively good 
yield of 2e may be caused by stabilization due to hyperconjugation of the 5-methyl group with the intermediary ethenyl carbocation. The reaction using silane $\mathbf{1 f}$ bearing a 1-hexynyl group rather than a trimethylsilylethynyl group did not afford the corresponding silole $\mathbf{2 f}$, and nearly all of $\mathbf{1 f}$ was recovered (entry 13). This result may be due to the intermediary ethenyl carbocation that was derived from $1 \mathbf{f}$ being less stable than those derived from 1a-e, which are stabilized by double $\beta$-silyl effects. Therefore, the trimethylsilyl group on the alkynyl group was essential for the current reaction.

Table 1. Intramolecular chain hydrosilylation of $\mathbf{1 a}-\mathbf{f}$ to $\mathbf{2 a}-\mathbf{f}$.

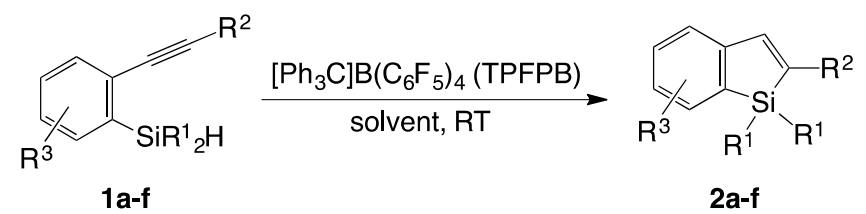

\begin{tabular}{|c|c|c|c|c|c|c|c|c|}
\hline Entry & & $\mathbf{R}^{1}$ & $\mathbf{R}^{2}$ & $\mathbf{R}^{3}$ & Solvent & TPFPB/mol \% & Time/min & Yield of $2 / \%^{a}$ \\
\hline 1 & 1a: & $\mathrm{Me}$ & TMS & $\mathrm{H}$ & benzene & 4 & 5 & $37(\mathbf{2 a})$ \\
\hline 2 & 1b: & $i-\operatorname{Pr}$ & TMS & $\mathrm{H}$ & benzene & 4 & 5 & $61(2 b)$ \\
\hline 3 & 1c: & $\mathrm{Ph}$ & TMS & $\mathrm{H}$ & benzene & 4 & 5 & $21(2 c)$ \\
\hline 4 & $1 b:$ & $i-\operatorname{Pr}$ & TMS & $\mathrm{H}$ & benzene & 3 & 15 & $70(\mathbf{2 b})$ \\
\hline 5 & $1 \mathrm{~b}:$ & $i-\operatorname{Pr}$ & TMS & $\mathrm{H}$ & benzene & 1 & 30 & $75(2 b)$ \\
\hline 6 & $1 b$ : & $i-\operatorname{Pr}$ & TMS & $\mathrm{H}$ & toluene & 1 & 30 & $72(2 b)$ \\
\hline 7 & $1 b:$ & $i-\operatorname{Pr}$ & TMS & $\mathrm{H}$ & mesitylene & 1 & 30 & $70(\mathbf{2 b})$ \\
\hline 8 & $1 b:$ & $i-\operatorname{Pr}$ & TMS & $\mathrm{H}$ & $\mathrm{CH}_{2} \mathrm{Cl}_{2}$ & 1 & 30 & $60(2 b)$ \\
\hline 9 & 1a: & $\mathrm{Me}$ & TMS & $\mathrm{H}$ & benzene & 1 & 30 & $34(2 a)$ \\
\hline 10 & 1c: & $\mathrm{Ph}$ & TMS & $\mathrm{H}$ & benzene & 1 & 30 & $55(2 c)$ \\
\hline 11 & 1d: & $i-\operatorname{Pr}$ & TMS & 4-Me & benzene & 1 & 30 & $72(2 d)$ \\
\hline 12 & 1e: & $i-\operatorname{Pr}$ & TMS & 5-Me & benzene & 1 & 50 & $81(2 \mathbf{e})$ \\
\hline 13 & 1f: & $i-\operatorname{Pr}$ & $n$-Bu & $\mathrm{H}$ & benzene & 1 & 720 & $0(\mathbf{2 f})^{b}$ \\
\hline
\end{tabular}

${ }^{\mathrm{a}}$ Isolated yields based on $\mathbf{1} ;{ }^{\mathrm{b}}$ Nearly all of $\mathbf{1 f}$ was recovered.

The reaction mechanism of the intramolecular chain hydrosilylation is described in Scheme 1. First, the trityl cation acts as an initiator to abstract the hydride from the $\mathrm{Si}-\mathrm{H}$ bond of $\mathbf{1}$, resulting in the generation of silyl cation A. Next, the intramolecular electrophilic addition of the silyl cation moiety of A to the C-C triple bond produces ethenyl carbocation $\mathbf{B}$, which is stabilized by the $\beta$-silyl effect of the trimethylsilyl group. Finally, the hydride abstraction of $\mathbf{B}$ from another $\mathbf{1}$ affords benzosilole $\mathbf{2}$ and regenerates intermediate $\mathbf{A}$ [27-29], which acts as a chain carrier.

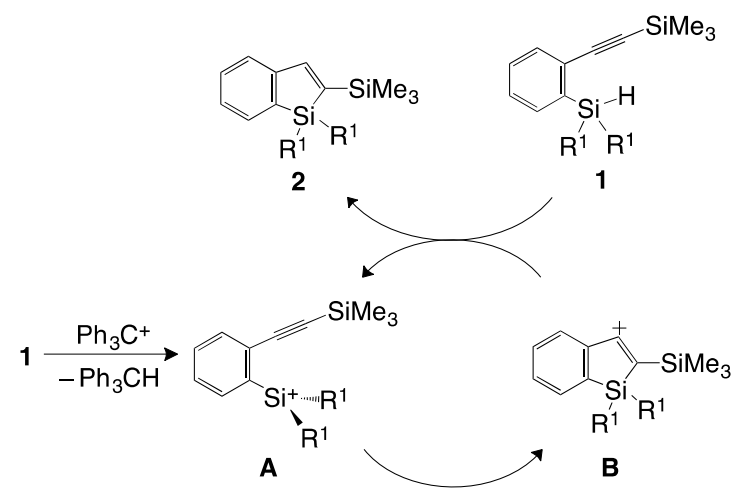

Scheme 1. Plausible mechanism of intramolecular chain hydrosilylation.

\section{Experimental Section}

General Procedure: All experiments were carried out using standard vacuum line and Schlenk techniques in an Ar atmosphere or dry box. All the reagents were of the highest grade available and were used without further purification. All solvents used for the syntheses were distilled 
according to the general procedure. $\left[\mathrm{Ph}_{3} \mathrm{C}\right] \mathrm{B}\left(\mathrm{C}_{6} \mathrm{~F}_{5}\right)_{4}$ [30], [2-(2-bromophenyl)ethynyl]trimethylsilane derivatives [31], 2-(1-hexynyl)bromobenzene [21], 1a [19] and 1c [21] were synthesized according to the previously reported methods. The NMR spectral measurements were performed on an Agilent 400-MR NMR (Agilent Technologies Co., Santa Clara, CA, USA) or a Bruker AV400M spectrometers (Bruker Co., Billerica, MA, USA). The ${ }^{1} \mathrm{H}$ and ${ }^{13} \mathrm{C}$ chemical shifts are reported relative to the residual protonated solvent and the solvent, respectively, according to the literature [32]. High-resolution mass spectrometry was measured by a JEOL GCMATE II (JEOL Ltd., Tokyo, Japan) or JMS-700N (JEOL Ltd.) operating by electron impact ionization (EI). Gel permeation liquid chromatography (GPLC) was performed by a Japan Analytical Industry LC-918 (Japan Analytical Industry Co., Ltd., Tokyo, Japan) using chloroform as an eluent.

\section{Preparation of Compounds}

Silanes 1. To a corresponding bromo compound $(1.3 \mathrm{mmol})$ in hexane $8 \mathrm{~mL}$ were added $1.6 \mathrm{M}$ pentane solution of tert-BuLi $(0.84 \mathrm{~mL}, 1.4 \mathrm{mmol})$ and $N, N, N^{\prime}, N^{\prime}$-tetramethylethylenediamine $(0.23 \mathrm{~g}$, $2.0 \mathrm{mmol}$ ) at $-80^{\circ} \mathrm{C}$, and the solution was stirred for $20 \mathrm{~min}$ keeping the temperature below $-70^{\circ} \mathrm{C}$. To the solution was added $i-\mathrm{Pr}_{2} \mathrm{SiHCl}(0.20 \mathrm{~g}, 1.4 \mathrm{mmol})$ at $-70{ }^{\circ} \mathrm{C}$, the solution was stirred and slowly warmed to room temperature. The reaction mixture was quenched with $5 \% \mathrm{NH}_{4} \mathrm{Cl}$ aqueous solution. The mixture was extracted with hexane $20 \mathrm{~mL}$ two times, and the organic layer was dried over anhydrous sodium sulfate. The filtrate was concentrated under reduced pressure to remove volatiles, and the residue was purified by silica gel column (eluent: hexane). Further purification was carried out by GPLC to obtain $\mathbf{1}$ as a colorless liquid.

Diisopropyl[(2-trimethylsilylethynyl)phenyl]silane (1b): 75\%. ${ }^{1} \mathrm{H}-\mathrm{NMR}\left(\mathrm{CDCl}_{3}, 400 \mathrm{MHz}\right): \delta 7.50-7.46$ $(\mathrm{m}, 2 \mathrm{H}, \mathrm{ArH}), 7.32-7.24(\mathrm{~m}, 2 \mathrm{H}, \mathrm{ArH}), 4.01(\mathrm{t}, J=4.0 \mathrm{~Hz}, 1 \mathrm{H}, \mathrm{SiH}), 1.49-1.39(\mathrm{~m}, 2 \mathrm{H}, i-\mathrm{Pr}), 1.10$ (d, $J=7.2 \mathrm{~Hz}, 6 \mathrm{H}, i-\mathrm{Pr}), 0.98$ (d, $J=7.6 \mathrm{~Hz}, 6 \mathrm{H}, i-\mathrm{Pr}), 0.24$ (s, 9H, $\left.\mathrm{SiMe}_{2}\right) .{ }^{13} \mathrm{C}-\mathrm{NMR}\left(\mathrm{CDCl}_{3}, 100 \mathrm{MHz}\right)$ : $\delta$ 138.1, 136.5, 132.6, 128.9, 128.8, 127.4, 106.6, 96.1, 19.12, 19.07, 11.1, -0.22. HRMS (EI) $m / z:[M]^{+}$Calcd for $\mathrm{C}_{17} \mathrm{H}_{28} \mathrm{Si}$, 288.1730; Found, 288.1729.

Diisopropyl[4-methyl-2-(trimethylsilylethynyl)phenyl]silane (1d): Yield 69\%. ${ }^{1} \mathrm{H}-\mathrm{NMR}\left(\mathrm{CDCl}_{3}, 400 \mathrm{MHz}\right)$ : $\delta 7.39(\mathrm{~d}, J=7.6 \mathrm{~Hz}, 1 \mathrm{H}, \mathrm{ArH}), 7.30(\mathrm{~d}, J=1.2 \mathrm{~Hz}, 1 \mathrm{H}, \mathrm{ArH}), 7.11(\mathrm{dd}, J=8.0 \mathrm{~Hz}, J=1.2 \mathrm{~Hz}, 1 \mathrm{H}, \mathrm{ArH})$, $3.98(\mathrm{t}, J=4.0 \mathrm{~Hz}, 1 \mathrm{H}, \mathrm{SiH}), 2.34(\mathrm{~s}, 3 \mathrm{H}, \mathrm{ArMe}), 1.50-1.40(\mathrm{~m}, 2 \mathrm{H}, i-\mathrm{Pr}), 1.11(\mathrm{~d}, J=7.6 \mathrm{~Hz}, 6 \mathrm{H}, i-\mathrm{Pr})$, $1.10(\mathrm{~d}, J=7.2 \mathrm{~Hz}, 6 \mathrm{H}, i-\mathrm{Pr}), 0.24$ (s, 9H, SiMe $) .{ }^{13} \mathrm{C}-\mathrm{NMR}\left(\mathrm{CDCl}_{3}, 100 \mathrm{MHz}\right): \delta 138.0,137.4,137.2$, 132.6, 129.6, 125.8, 106.9, 95.1, 21.5, 19.20, 19.13, 11.2, -0.18. HRMS (EI) $m / z$ : [M] $]^{+}$Calcd for $\mathrm{C}_{18} \mathrm{H}_{30} \mathrm{Si}_{2}$, 302.1886; Found, 302.1909.

Diisopropyl[5-methyl-2-(trimethylsilylethynyl)phenyl]silane (1e): 68\%. ${ }^{1} \mathrm{H}-\mathrm{NMR}\left(\mathrm{CDCl}_{3}, 400 \mathrm{MHz}\right): \delta 7.46$ $(\mathrm{dd}, J=7.2 \mathrm{~Hz}, J=1.2 \mathrm{~Hz}, 1 \mathrm{H}, \mathrm{ArH}), 7.41(\mathrm{~d}, J=7.2 \mathrm{~Hz}, 1 \mathrm{H}, \mathrm{ArH}), 7.28(\mathrm{td}, J=7.6 \mathrm{~Hz}, J=1.6 \mathrm{~Hz}, 1 \mathrm{H}$, $\mathrm{ArH}), 7.22(\mathrm{td}, J=7.6 \mathrm{~Hz}, J=1.6 \mathrm{~Hz}, 1 \mathrm{H}, \mathrm{ArH}), 4.02(\mathrm{t}, J=4.0 \mathrm{~Hz}, 1 \mathrm{H}, \mathrm{SiH}), 2.41(\mathrm{t}, J=7.2 \mathrm{~Hz}, 2 \mathrm{H}$, $n-\mathrm{Bu}), 1.62-1.32(\mathrm{~m}, 6 \mathrm{H}, i-\mathrm{Pr}$ and $n-\mathrm{Bu}), 1.10(\mathrm{~d}, J=7.6 \mathrm{~Hz}, 6 \mathrm{H}, i-\mathrm{Pr}), 0.98(\mathrm{~d}, J=7.2 \mathrm{~Hz}, 6 \mathrm{H}, i-\mathrm{Pr}), 0.95$ $\left(\mathrm{t}, J=7.2 \mathrm{~Hz}, 3 \mathrm{H}, n\right.$-Bu). ${ }^{13} \mathrm{C}-\mathrm{NMR}\left(\mathrm{CDCl}_{3}, 100 \mathrm{MHz}\right): \delta 137.4,136.4,132.1,130.0,128.8,126.4,92.4,82.1$, 30.7, 22.1, 19.24, 19.12, 13.6, 11.1. HRMS (EI) $\mathrm{m} / \mathrm{z}$ : [M] ${ }^{+}$Calcd for $\mathrm{C}_{18} \mathrm{H}_{28} \mathrm{Si}$, 272.1960; Found, 272.1958 .

[2-(1-Hexynyll)phenyl]diisopropylsilane (1f): Yield 55\%. ${ }^{1} \mathrm{H}-\mathrm{NMR}\left(\mathrm{CDCl}_{3}, 400 \mathrm{MHz}\right): \delta 7.38(\mathrm{~d}, J=7.6 \mathrm{~Hz}$, 1H, ArH), $7.34(\mathrm{~s}, 1 \mathrm{H}, \mathrm{ArH}), 7.10(\mathrm{~d}, J=7.6 \mathrm{~Hz}, 1 \mathrm{H}, \mathrm{ArH}), 4.00(\mathrm{t}, J=4.0 \mathrm{~Hz}, 1 \mathrm{H}, \mathrm{SiH}), 2.31(\mathrm{~s}, 3 \mathrm{H}$, ArMe), $1.47-1.36$ (m, 2H, i-Pr), 1.10 (d, $J=7.2 \mathrm{~Hz}, 6 \mathrm{H}, i-\operatorname{Pr}), 0.98$ (d, $J=7.2 \mathrm{~Hz}, 6 \mathrm{H}, i-\mathrm{Pr}), 0.24$ (s, 9H, $\left.\mathrm{SiMe}_{3}\right) .{ }^{13} \mathrm{C}-\mathrm{NMR}\left(\mathrm{CDCl}_{3}, 100 \mathrm{MHz}\right): \delta 138.7,136.7,134.4,133.3,128.8,128.5,106.8,95.6,21.1,19.12$, 19.09, 11.1, - 0.21. HRMS (EI) $m / z$ : [M] ${ }^{+}$Calcd for $\mathrm{C}_{18} \mathrm{H}_{30} \mathrm{Si}_{2}, 302.1886$; Found, 302.1883.

Benzosiloles 2. To trityl tetrakis(pentafluorophenyl)borate (TPFPB, $1.0 \mathrm{mg}, 1.0 \mu \mathrm{mol}$ ) in benzene $(0.5 \mathrm{~mL})$ was added a benzene solution $(1.5 \mathrm{~mL})$ of hydrosilanes $\mathbf{1}(0.10 \mathrm{mmol})$ at room temperature under Ar atmosphere, and the resulting solution was stirred at room temperature. After the reaction 
mixture was quenched with 2,6-lutidine $(2 \mu \mathrm{L})$ and $\mathrm{H}_{2} \mathrm{O}$, and then the organic layer was extracted. After extraction with hexane two times, the organic layers were combined and dried over anhydrous sodium sulfate, and then the filtrate was evaporated under reduced pressure to remove volatiles. Purification was carried out by GPLC to remove polymeric materials.

1,1-Dimethyl-2-trimethylsilyl-1-silaindene (2a): 34\%. ${ }^{1} \mathrm{H}-\mathrm{NMR}$ data are consisted with those reported previously [19].

1,1-Diisopropyl-2-trimethylsilyl-1-silaindene (2b): 75\%. ${ }^{1} \mathrm{H}-\mathrm{NMR}\left(\mathrm{CDCl}_{3}, 400 \mathrm{MHz}\right): \delta 7.61(\mathrm{~s}, 1 \mathrm{H}$, $\mathrm{ArCH}=\mathrm{C}), 7.51(\mathrm{~d}, J=6.8 \mathrm{~Hz}, 2 \mathrm{H}, \mathrm{ArH}), 7.32(\mathrm{td}, J=7.6 \mathrm{~Hz}, J=1.2 \mathrm{~Hz}, 1 \mathrm{H}, \mathrm{ArH}), 7.27(\mathrm{~d}, J=6.4 \mathrm{~Hz}, 1 \mathrm{H}$, $\mathrm{ArH}), 7.19(\mathrm{td}, J=7.6 \mathrm{~Hz}, J=1.2 \mathrm{~Hz}, 1 \mathrm{H}, \mathrm{ArH}), 1.35-1.25(\mathrm{~m}, 2 \mathrm{H}, i-\mathrm{Pr}), 1.07(\mathrm{~d}, J=7.6 \mathrm{~Hz}, 6 \mathrm{H}, i-\mathrm{Pr}), 0.93$ (d, $J=7.6 \mathrm{~Hz}, 6 \mathrm{H}, i-\mathrm{Pr}), 0.19$ (s, 9H, $\left.\mathrm{SiMe}_{2}\right) .{ }^{13} \mathrm{C}-\mathrm{NMR}\left(\mathrm{CDCl}_{3}, 100 \mathrm{MHz}\right): \delta 158.2,151.1,143.1,137.4$, 132.6, 129.4, 126.7, 123.9, 19.98, 19.96, 11.2, -0.16. HRMS (EI) $m / z$ : [M] ${ }^{+}$Calcd for $\mathrm{C}_{17} \mathrm{H}_{28} \mathrm{Si}, 288.1730$; Found, 288.1736.

1,1-Diphenyl-2-trimethylsilyl-1-silaindene (2c): 55\%. ${ }^{1} \mathrm{H}-\mathrm{NMR}$ data are consisted with those reported previously [21].

1,1-Diisopropyl-5-methyl-2-trimethylsilyl-1-silaindene (2d): Yield 72\%. ${ }^{1} \mathrm{H}-\mathrm{NMR}\left(\mathrm{CDCl}_{3}, 400 \mathrm{MHz}\right): \delta 7.57$ $(\mathrm{s}, 1 \mathrm{H}, \mathrm{ArCH}=\mathrm{C}), 7.40(\mathrm{~d}, J=6.8 \mathrm{~Hz}, 2 \mathrm{H}, \mathrm{ArH}), 7.11(\mathrm{~s}, 1 \mathrm{H}, \mathrm{ArH}), 7.02(\mathrm{~d}, J=6.8 \mathrm{~Hz}, 1 \mathrm{H}, \mathrm{ArH}), 2.35$ (s, 3H, ArMe), 1.34-1.22 (m, 2H, i-Pr), 1.06 (d, J = 7.2 Hz, 6H, i-Pr), $0.93(\mathrm{~d}, J=7.2 \mathrm{~Hz}, 6 \mathrm{H}, i-\mathrm{Pr}), 0.19$ (s, 9H, $\left.\mathrm{SiMe}_{2}\right) .{ }^{13} \mathrm{C}-\mathrm{NMR}\left(\mathrm{CDCl}_{3}, 100 \mathrm{MHz}\right): \delta 158.2,151.6,143.3,139.3,133.6,132.5,127.5,125.0,21.5$, 18.01, 17.99, 11.2, -0.16. HRMS (EI) $\mathrm{m} / z$ : [M] ${ }^{+}$Calcd for $\mathrm{C}_{18} \mathrm{H}_{30} \mathrm{Si}_{2}, 302.1886$; Found, 302.1911.

1,1-Diisopropyl-6-methyl-2-trimethylsilyl-1-silaindene (2e): Yield 81\%. ${ }^{1} \mathrm{H}-\mathrm{NMR}\left(\mathrm{CDCl}_{3}, 400 \mathrm{MHz}\right): \delta 7.58$ (s, 1H, ArH), 7.32 (s, 1H, ArH), 7.18-7.09 (m, 2H, ArH), 2.36 (s, 3H, ArMe), 1.36-1.22 (m, 2H, i-Pr), 1.07 $(\mathrm{d}, J=7.2 \mathrm{~Hz}, 6 \mathrm{H}, i-\mathrm{Pr}), 0.94(\mathrm{~d}, J=7.6 \mathrm{~Hz}, 6 \mathrm{H}, i-\mathrm{Pr}), 0.19$ (s, 9H, SiMe $) .{ }^{13} \mathrm{C}-\mathrm{NMR}\left(\mathrm{CDCl}_{3}, 100 \mathrm{MHz}\right)$ : $\delta 158.0,148.7,141.3,137.6,136.2,133.6,130.0,123.6,21.4,17.98,17.9,11.2,-0.13$. HRMS (EI) $\mathrm{m} / z:[\mathrm{M}]^{+}$ Calcd for $\mathrm{C}_{18} \mathrm{H}_{30} \mathrm{Si}_{2}, 302.1886$; Found, 302.1863.

The ${ }^{1} \mathrm{H}$ - and ${ }^{13} \mathrm{C}-\mathrm{NMR}$ spectral charts of all new compounds $\mathbf{1}$ and $\mathbf{2}$ are summarized in supplementary materials.

\section{Conclusions}

In conclusions, we achieved the intramolecular chain hydrosilylation of $\mathbf{1}$ to synthesize benzosiloles 2 . The hydrosilylation proceeded under mild conditions with a small amount of TPFPB as an initiator and no additives. In this reaction, the silyl cation plays an important role as a chain carrier, which is different from the reactions involving the hydridosilicate and silyl radical.

Supplementary Materials: The ${ }^{1} \mathrm{H}$ - and ${ }^{13} \mathrm{C}-\mathrm{NMR}$ spectral charts of $\mathbf{1 b}, \mathbf{1 d}, \mathbf{1 e}, \mathbf{1 f}, \mathbf{2} \mathbf{b}, \mathbf{2} \mathbf{d}$, and $\mathbf{2 e}$ can be accessed at http:/ /www.mdpi.com/1420-3049/21/8/999/s1.

Acknowledgments: This work was financially supported by a Grant-in-Aid for Scientific Research (No. 26410035, T.K.) from the Ministry of Education, Culture, Sports, Science and Technology, Japan. This work was performed under the Cooperative Research Program of "Network Joint Research Center for Materials and Devices" (H.A.). We acknowledge the Center for Collaborative Research and Community Cooperation (Miyazaki University) for the NMR spectroscopy and elemental analysis. We wish to thank Nobuaki Tsuda (Center for Industry, University and Government Cooperation, Nagasaki University) for measurement of the high-resolution mass spectroscopy.

Author Contributions: H.A. conceived and performed the experiments. T.K. designed the present reaction. H.A., K.N., K.M. and T.K. discussed the results. H.A. wrote the manuscript and T.K. edited it.

Conflicts of Interest: The authors declare no conflict of interest. 


\section{References and Notes}

1. Yamaguchi, S.; Tamao, K. Silole-containing $\sigma$ - and $\pi$-conjugated compounds. J. Chem. Soc. Dalton Trans 1998, 27, 3693-3702. [CrossRef]

2. Wong, W.W.H.; Holms, A.B. Poly(dibenzosilole)s. Adv. Polym. Sci. 2008, 212, 85-98.

3. Chen, J.; Cao, Y. Development of novel conjugated donor polymers for high-efficiency bulk-heterojunction photovoltaic devices. Acc. Chem. Res. 2009, 42, 1709-1718. [CrossRef] [PubMed]

4. Facchetti, A. $\pi$-Conjugated polymers for organic electronics and photovoltaic cell applications. Chem. Mater. 2011, 23, 733-758. [CrossRef]

5. Yamaguchi, S.; Tamao, K. Theoretical study of the electronic structure of 2,2'-bisilole in comparison with 1,1'-bi-1,3-cyclopentadiene: $\sigma^{*}-\pi^{*}$ Conjugation and a low-lying LUMO as the origin of the unusual optical properties of 3,3'4,4'-tetraphenyl-2,2'-bisilole. Bull. Chem. Soc. Jpn. 1996, 69, 2327-2334. [CrossRef]

6. Shimizu, M.; Mochida, K.; Hiyama, T. Modular approach to silicon-bridged biaryls: Palladium-catalyzed intramolecular coupling of 2-(arylsilyl)aryl triflates. Angew. Chem. Int. Ed. 2008, 47, 9760-9764. [CrossRef] [PubMed]

7. Tobisu, M.; Onoe, M.; Kita, Y.; Chatani, N. Rhodium-catalyzed coupling of 2-silylphenylboronic acids with alkynes leading to benzosiloles: Catalytic cleavage of the carbon-silicon bond in trialkylsilyl groups. J. Am. Chem. Soc. 2009, 131, 7506-7507. [CrossRef] [PubMed]

8. Ouyang, K.; Liang, Y.; Xi, Z. Construction of benzosiloles, six- and eight-membered silacyclic skeletons via a Pd-catalyzed intramolecular Mizoroki-Heck reaction of vinylsilanes. Org. Lett. 2012, 14, 4572-4575. [CrossRef] [PubMed]

9. Onoe, M.; Baba, K.; Kim, Y.; Kita, Y.; Tobisu, M.; Chatani, N. Rhodium-catalyzed carbon-silicon bond activation for synthesis of benzosilole derivatives. J. Am. Chem. Soc. 2012, 134, 19477-19488. [CrossRef] [PubMed]

10. Shintani, R.; Otomo, H.; Ota, K.; Hayashi, T. Palladium-catalyzed asymmetric synthesis of silicon-stereogenic dibenzosiloles via enantioselective C-H bond functionalization. J. Am. Chem. Soc. 2012, 134, 7305-7308. [CrossRef] [PubMed]

11. Shintani, R.; Takagi, C.; Ito, T.; Naito, M.; Nozaki, K. Rhodium-catalyzed asymmetric synthesis of silicon-stereogenic dibenzosiloles by enantioselective $[2+2+2]$ cycloaddition. Angew. Chem. Int. Ed. 2015, 54, 1616-1620. [CrossRef] [PubMed]

12. Matsuda, T.; Kadowaki, S.; Murakami, M. Ruthenium-catalyzed double trans-hydrosilylation of 1,4-diarylbuta-1,3-diynes leading to 2,5-diarylsiloles. Chem. Commun. 2007, 43, 2627-2629. [CrossRef] [PubMed]

13. Ureshino, T.; Yoshida, T.; Kuninobu, Y.; Takai, K. Rhodium-catalyzed synthesis of silafluorene derivatives via cleavage of silicon-hydrogen and carbon-hydrogen bonds. J. Am. Chem. Soc. 2010, 132, 14324-14326. [CrossRef] [PubMed]

14. Matsuda, T.; Yamaguchi, Y.; Shigeno, M.; Sato, S.; Murakami, M. Gold-catalysed alkenyl- and arylsilylaton reactions forming 1-silaindenes. Chem. Commun. 2011, 47, 8697-8699. [CrossRef] [PubMed]

15. Zhang, Q.-W.; An, K.; He, W. Rhodium-catalyzed tandem cyclization/Si-C activation reaction for the synthesis of siloles. Angew. Chem. Int. Ed. 2014, 53, 5667-5671. [CrossRef] [PubMed]

16. Omann, L.; Oestreich, M. A catalytic $S_{E}$ Ar approach to dibenzosiloles functionalized at both benzene cores. Angew. Chem. Int. Ed. 2015, 54, 10276-10279. [CrossRef] [PubMed]

17. Zhang, Q.-W.; An, K.; Liu, L.-C.; Guo, S.; Jiang, C.; Guo, H.; He, W. Rhodium-catalyzed intramolecular C-H silylation by silacyclobutanes. Angew. Chem. Int. Ed. 2016, 55, 6319-6323. [CrossRef] [PubMed]

18. Xu, C.; Wakamiya, A.; Yamaguchi, S. General silaindene synthesis based on intramolecular reductive cyclization toward new fluorescent silicon-containing $\pi$-electron materials. Org. Lett. 2004, 6, 3707-3710. [CrossRef] [PubMed]

19. Sudo, T.; Asao, N.; Yamamoto, Y. Synthesis of various silacycles via the Lewis acid-catalyzed intramolecular trans-hydrosilylation of unactivated alkynes. J. Org. Chem. 2000, 65, 8919-8923. [CrossRef] [PubMed]

20. Curless, L.D.; Ingleson, M.J. B $\left(\mathrm{C}_{6} \mathrm{~F}_{5}\right)_{3}$-catalyzed synthesis of benzofused-siloles. Organometallics 2014, 33, 7241-7246. [CrossRef]

21. Ilies, L.; Tsuji, H.; Nakamura, E. Synthesis of benzo[b]siloles via KH-promoted cyclization of (2-alkynylphenyl)silanes. Org. Lett. 2009, 11, 3966-3968. [CrossRef] [PubMed] 
22. Leifert, D.; Studer, A. 9-Silafluorenes via base-promoted homolytic aromatic substitution (BHAS)-The electron as catalyst. Org. Lett. 2015, 17, 386-389. [CrossRef] [PubMed]

23. Furukawa, S.; Kobayashi, J.; Kawashima, T. Development of a sila-Friedel-Crafts reaction and its application to the synthesis of dibenzosilole derivatives. J. Am. Chem. Soc. 2009, 131, 14192-14193. [CrossRef] [PubMed]

24. Furukawa, S.; Kobayashi, J.; Kawashima, T. Application of sila-Friedel-Crafts reaction to the synthesis of $\pi$-extended silole derivatives and their properties. Dalton Trans. 2010, 39, 9329-9336. [CrossRef] [PubMed]

25. Arii, H.; Kurihara, T.; Mochida, K.; Kawashima, T. Silylium ion-promoted dehydrogenative cyclization: Synthesis of silicon-containing compounds derived from alkynes. Chem. Commun. 2014, 50, 6649-6652. [CrossRef] [PubMed]

26. Synthesis of silacycles using alkenes instead of alkynes: see, Arii, H.; Yano, Y.; Nakabayashi, K.; Yamaguchi, S.; Yamamura, M.; Mochida, K.; Kawashima, T. Regioselective and stereospecific dehydrogenative annulation utilizing silylium ion-activated alkenes. J. Org. Chem. 2016. [CrossRef]

27. Lambert, J.B.; Zhao, Y.; Wu, H. $\beta$-Silyl and $\beta$-germyl carbocations stable at room temperature. J. Org. Chem. 1999, 64, 2729-2736. See similar self-regeneration of silyl cations in [28,29].

28. Müther, K.; Oestreich, M. Self-regeneration of a silylium ion catalyst in carbonyl reduction. Chem. Commun. 2011, 47, 334-336. See similar self-regeneration of silyl cations in [27,29].

29. Müther, K.; Mohr, J.; Oestreich, M. Silylium ion promoted reduction of imines with hydrosilanes. Organometallics 2013, 32, 6643-6646. See similar self-regeneration of silyl cations in [27,28].

30. Ihara, E.; Young, V.G., Jr.; Jordan, R.F. Cationic aluminium alkyl complexes incorporating aminotroponiminate ligands. J. Am. Chem. Soc. 1998, 120, 8277-8278. [CrossRef]

31. Matsuda, T.; Kato, K.; Goya, T.; Shimada, S.; Murakami, M. Ruthenium-catalyzed cycloisomerization of 2,2'-diethynylbiphenyls involving cleavage of a carbon-carbon triple bond. Chem. Eur. J. 2016, 22, 1941-1943. [CrossRef] [PubMed]

32. Fulmer, G.R.; Miller, A.J.M.; Sherden, N.H.; Gottlieb, H.E.; Nuldelman, A.; Stoltz, B.M.; Bercaw, J.E.; Goldberg, K.I. NMR chemical shifts of trace impurities: Common laboratory solvents, organics, and gases in deuterated solvents relevant to the organometallic chemist. Organometallics 2010, 29, 2176-2179. [CrossRef]

Sample Availability: Samples of the compounds 1 and $\mathbf{2}$ are available from the authors.

(c) 2016 by the authors; licensee MDPI, Basel, Switzerland. This article is an open access article distributed under the terms and conditions of the Creative Commons Attribution (CC-BY) license (http://creativecommons.org/licenses/by/4.0/). 